\title{
Search for single-nucleotide polymorphisms associated with accelerated senescence in OXYS rats
}

\author{
Devyatkin V. ${ }^{1,2 *}$, Redina O. ${ }^{1}$, Muraleva N. ${ }^{1}$, Kolosova N. ${ }^{1}$ \\ ${ }^{1}$ Institute of Cytology and Genetics, SB RAS, Novosibirsk, Russia \\ ${ }^{2}$ Novosibirsk State University, Novosibirsk, Russia \\ *e-mail:devyatkin@bionet.nsc.ru
}

Key words: aging, age-related diseases, OXYS rats, SNPs

Motivation and Aim: Aging is the largest risk factor for many diseases. However, little is known about a genetic overlap between age-related diseases. The senescenceaccelerated OXYS rats selected in the ICG SB RAS (Novosibirsk) are a unique model to identify the pathways that modulate the onset and progression of multiple age-related diseases as these rats develop a phenotype similar to human geriatric disorders including cataract, cardiomyopathy, hypertension, retinopathy and neurodegenerative pathology of the brain with features of Alzheimer's disease. The aim of our work was to investigate the transcriptome of OXYS rats and to identify the mutations (SNPs) in genes, which can potentially contribute to the development of accelerated aging.

Methods and Algorithms: The RNA-Seq data were obtained from sequencing of prefrontal cortex, retina and hippocampus of senescence-accelerated OXYS rats. Positions of SNPs within the aligned reads relative to the reference genome (Rnor 6.0) were identified using SAMtools (v. 0.1.17) utilities. The mutation was considered as reliable SNP if it was detected in at least 3 OXYS rats in homozygous state and coverage (DP) was $\geq$ 10 in at least one animal. The effect of an amino acid substitution on protein function was predicted by the Variant Effect Predictor Web service (https:/www.ensembl.org/ vep); the consequence type, SIFT score and prediction were obtained for each variant. The SNPs found in OXYS rats were compared with the data of genomic sequencing of 42 other rat strains, 11 of which simulate different forms of hypertension, 10 strains are used as a normotensive control, and the rest are used as experimental or control strains to study diseases not related to hypertension or aging.

Results: In the genome of OXYS rats 42478 SNPs overlapping with 9903 genes were detected. Of these, 725 SNPs were found both in OXYS rats and in one or several other hypertensive rat strains, and 1380 SNPs were specific to OXYS rats. These 2105 SNPs can contribute to the development of the accelerated aging phenotype; of these, 9 SNPs can lead to significant structural rearrangements of transcripts, and 33 SNPs probably have a significant effect on the structure or function of the protein products. Several of these SNPs are mapped to genes associated with mitochondrial and neurodegenerative diseases, mental and cardiovascular disorders, which is consistent with the complex manifestation of the senile phenotype in OXYS rats.

Conclusion: The results of the study may serve as a background for further verification of SNPs contribution to the development of complex age-related diseases in OXYS and other rat strains as well as in conducting GWAS in humans.

Acknowledgements: Supported by the RFBR (project No. 18-015-00336, No. 18-31500216). 